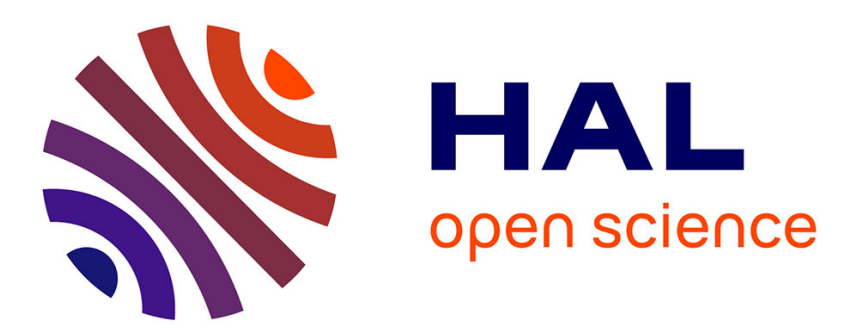

\title{
Theory and experiments on multistep parametric processes in nonlinear optics
}

Matteo Conforti, F. Baronio, C. de Angelis, M. Marangoni, G. Cerullo

\section{To cite this version:}

Matteo Conforti, F. Baronio, C. de Angelis, M. Marangoni, G. Cerullo. Theory and experiments on multistep parametric processes in nonlinear optics. Journal of the Optical Society of America B, 2011, 28 (4), pp.892. 10.1364/JOSAB.28.000892 . hal-02395360

\section{HAL Id: hal-02395360 \\ https://hal.science/hal-02395360}

Submitted on 5 Dec 2019

HAL is a multi-disciplinary open access archive for the deposit and dissemination of scientific research documents, whether they are published or not. The documents may come from teaching and research institutions in France or abroad, or from public or private research centers.
L'archive ouverte pluridisciplinaire HAL, est destinée au dépôt et à la diffusion de documents scientifiques de niveau recherche, publiés ou non, émanant des établissements d'enseignement et de recherche français ou étrangers, des laboratoires publics ou privés. 


\section{Theory and experiments on multistep parametric processes in}

\section{nonlinear optics}

\section{Conforti, ${ }^{1}$ F. Baronio, ${ }^{1}$ C. De Angelis, ${ }^{1}$ M. Marangoni, ${ }^{2}$ and G. Cerullo ${ }^{2}$}

${ }^{1}$ CNISM, Dipartimento di Ingegneria dell'Informazione, Università di Brescia, Via Branze 38, 25123 Brescia, Italy

e-mail: matteo.conforti@ing.unibs.it

${ }^{2}$ IFN-CNR, Dipartimento di Fisica, Politecnico di Milano,

Piazza Leonardo da Vinci 32, 20133 Milano, Italy

We present a comprehensive model for the description of different types of parametric interactions, associated with simultaneous phase-matching of several optical processes: the so-called multistep parametric interactions. Our approach is based on a recently derived single wave broadband equation that is able to describe general quadratic nonlinear optical interactions and can be solved with modest computational effort. We compare theoretical results with experiments on simultaneous second and third harmonic generation performed in periodically poled lithium tantalate crystals.

(C) 2011 Optical Society of America

OCIS codes: $190.2620,190.4223,320.2250$. 


\section{Introduction}

Energy transfer between waves oscillating at different frequencies is one of the fundamental concepts in nonlinear optics and has been studied intensively since the early 1960s [1]. Parametric processes naturally take place in nonlinear materials where the lowest-order nonlinear contribution to the polarization of the medium is quadratic in the electric field $\left(P \propto \chi^{(2)} E^{2}\right)$ and involve several waves at different frequencies [2]. In order to be efficient the parametric coupling requires special relations between the phase velocities of the interacting waves to be satisfied, the so called phase matching conditions. Phase matching is either realized exactly by birefringence, or by periodically reversing the sign of the nonlinear coefficient $\chi^{(2)}$ by quasi phase matching (QPM). The QPM technology permits also to engineer the nonlinear response of the medium and to obtain "nonlinear metamaterials" where the quadratic properties can be designed at will $[3,4]$.

Multistep parametric interactions [5] represent a special type of second-order parametric process that involves several different second-order nonlinear interactions and is characterized by at least two different phase matching parameters. For example it is possible to obtain cascaded third harmonic generation (THG) from two concurrent processes: second harmonic generation (SHG) $\omega+\omega=2 \omega$ and sum frequency mixing (SFM) $\omega+2 \omega=3 \omega$.

There exists a wide variety of multistep parametric processes, such as third harmonic generation [6], two-color parametric interaction [7], fourth harmonic generation [8], wavelength conversion [9] and many others [5]. In general it is hard to phase match different processes by traditional methods such as birefringence phase matching, but QPM structures with chirped and aperiodic gratings supply the requested flexibility.

This variety of phenomena requires a variety of mathematical models, that are usually derived in the frame of coupled slowly varying field envelopes. These models consist of 
$N$ equations for the envelopes $A_{i}, i=1, \ldots, N$ which are nonlinearly coupled through quadratic nonlinear terms such as $A_{n}^{2}, A_{m} A_{n}$ or $A_{m} A_{n}^{*}$ (in Ref. [5] a non exhaustive list of the most frequent cases is reported). It is clear that a deep knowledge of the physical setting is required in order to select the correct model, and all the matched processes have to be considered, in order to obtain consistent results: for example in a periodically poled lithium niobate (PPLN) sample several spatial harmonics of the grating can match several different processes. Sometimes the interacting waves have bandwidths that can be comparable with their frequency separation, making the division into harmonics somewhat arbitrary . For example one can ask if in a SFM process $\omega_{1}+\omega_{2}=\omega_{3}$, where $\omega_{1}$ and $\omega_{2}$ have a frequency separation comparable with their bandwidth, is more correct to model the process as a two or three wave process.

A different approach to the study of multistep quadratic phenomena is to exploit a single nonlinear envelope equation (NEE) for the entire frequency interval involved in the process. Such a nonlinear envelope equation was derived recently and was shown to be accurate to model several quadratic processes $[10,11]$. This model is particularly suited for the purpose of numerically simulating the optical pulse propagation: in fact the computational resources required for the solution of this model are of the order of magnitude of those required for standard coupled wave equations.

In this article we show how to exploit the NEE for the description of multistep parametric processes. We show that a single wave equation can describe with great accuracy phenomena spanning several optical octaves. We also show that the relevant coupled wave models can be derived directly from the NEE with minimal assumptions.

The rest of the paper is organized as follows: in Section 2 we describe the single wave model and we outline the derivation of the the three wave equation describing cascading 
THG. In Section 3 we compare numerical and experimental results of simultaneous second and third harmonic generation in a periodically poled lithium tantalate crystal (PPLT). Finally we present out conclusions in section 4.

\section{Nonlinear $\chi^{(2)}$ envelope equation}

The starting point of our analysis is the following equation $[10,11]$ :

$$
\begin{array}{r}
\frac{\partial A}{\partial z}+i D A=-i G(z) \frac{\chi^{(2)} \omega_{0}}{4 n_{0} c}\left(1-\frac{i}{\omega_{0}} \frac{\partial}{\partial t}\right)\left[A^{2} e^{i \omega_{0} t-i \beta_{0} z}\right. \\
\left.+2|A|^{2} e^{-i \omega_{0} t+i \beta_{0} z}\right]
\end{array}
$$

where $c$ is the velocity of light in vacuum, $\hat{\varepsilon}(\omega)=1+\hat{\chi}(\omega), \hat{\chi}(\omega)$ is the linear electric

susceptibility and $k(\omega)=(\omega / c) \sqrt{\hat{\varepsilon}(\omega)}$ is the propagation constant, $A=A(z, t)$ is the electric field envelope as defined in [10], $\omega_{0}$ is a reference frequency, $n_{0}=n\left(\omega_{0}\right)$ is the reference refractive index, $\beta_{0}=k\left(\omega_{0}\right)$ is the reference wavevector, $\chi^{(2)}$ is the nonlinear coefficient and $G(z)$ describes the spatial modulation of the nonlinear material. The dispersive operator is defined as $D=\sum_{m=1}^{\infty} \frac{1}{m !} k_{m}\left(-i \frac{\partial}{\partial t}\right)^{m}$ where $k_{m}=\frac{\partial^{m} k}{\partial \omega^{m}}\left(\omega_{0}\right)$. It is possible, if needed, to consider other and more general expressions of the dispersive operator [12].

The nonlinear envelope equation (1), first order in propagation coordinate, provides a powerful means of describing light pulse propagation in dispersive quadratically nonlinear media. Using an approach exploited in [13] for cubic nonlinear media, equation (1) was first reported in [10], then derived rigorously extending its range of validity in [11] . Equation (1) can be solved easily by split-step Fourier method exploiting the exact frequency dependence of the propagation constant for the linear step, and a fourth order Runge-Kutta scheme for the nonlinear step.

It has been pointed out that evolution equations like (1) describe unidirectional elec- 
tromagnetic propagation with a surprising degree of accuracy, almost without restrictions on the frequency extent of the signals $[10,13-15]$ : in this sense they can be considered as forward Maxwell equations [16].

In order to show the validity of Eq. (1) over a bandwidth of several optical octaves and its ability to model multistep parametric processes, we derive from (1) the three-wave model for the description of cascading third harmonic generation $[5,17]$. We consider an electric field envelope composed of three waves:

$$
\begin{aligned}
A(z, t) & =A_{1}(z, t) e^{i\left(\Omega-\omega_{0}\right) t-i\left(q_{1}-\beta_{0}\right) z} \\
& +A_{2}(z, t) e^{i\left(2 \Omega-\omega_{0}\right) t-i\left(q_{2}-\beta_{0}\right) z} \\
& +A_{3}(z, t) e^{i\left(3 \Omega-\omega_{0}\right) t-i\left(q_{3}-\beta_{0}\right) z}
\end{aligned}
$$

where $\Omega$ is the fundamental frequency and $q_{1}, q_{2}, q_{3}$ are the propagation constants at fundamental, second and third harmonic.

In order to handle the dispersive term in a straightforward manner we first Fourier transform the Eq. (1):

$$
\frac{\partial \hat{A}(\omega)}{\partial z}+i\left[k\left(\omega+\omega_{0}\right)-k_{0}\right] \hat{A}(\omega)=N L
$$

where $N L$ stands for the Fourier transform of the nonlinear terms. The difference in the frequency dependence between $\hat{A}($.$) and k($.$) is given by the fact that A$ is the envelope and is centered around zero in frequency domain, whereas the wavenumber $k$ has the same dependence of the total electric field, that is centered around $\omega_{0}$. It must be kept in mind that this frequency offset is totally arbitrary and it is not assumed that the band of the envelope must be narrow with respect to $\omega_{0}[10]$.

Now inserting (Fourier transform of) ansatz (2) in (3) we obtain:

$$
\sum_{m=1}^{3}\left[\frac{\partial \hat{A}_{m}}{\partial z}\left(\omega-\left(m \Omega-\omega_{0}\right)\right)+\right.
$$




$$
\left.i\left[k\left(\omega+\omega_{0}\right)-q_{m}\right] \hat{A}_{m}\left(\omega-\left(m \Omega-\omega_{0}\right)\right)\right] e^{-i\left(q_{m}-\beta_{0}\right) z}=N L
$$

that after inverse Fourier transform reads:

$$
\begin{array}{r}
\sum_{m=1}^{3}\left[\frac{\partial A_{m}}{\partial z}+i D_{m} A_{m}\right] e^{i\left(m \Omega-\omega_{0}\right)-i\left(q_{m}-\beta_{0}\right) z}= \\
-i G(z) \frac{\chi^{(2)} \omega_{0}}{4 n_{0} c}\left(1-\frac{i}{\omega_{0}} \frac{\partial}{\partial t}\right)\left[A_{1}^{2} e^{i\left(2 \Omega-\omega_{0}\right) t-i\left(2 q_{1}-\beta_{0}\right) z}\right. \\
+2 A_{1} A_{2} e^{i\left(3 \Omega-\omega_{0}\right) t-i\left(q_{1}+q_{2}-\beta_{0}\right) z} \\
+2 A_{2} A_{1}^{*} e^{i\left(\Omega-\omega_{0}\right) t-i\left(q_{2}-q_{1}-\beta_{0}\right) z} \\
+2 A_{3} A_{2}^{*} e^{i\left(\Omega-\omega_{0}\right) t-i\left(q_{3}-q_{1}-\beta_{0}\right) z} \\
\left.+2 A_{3} A_{1}^{*} e^{i\left(2 \Omega-\omega_{0}\right) t-i\left(q_{3}-q_{1}-\beta_{0}\right) z}+R\right]
\end{array}
$$

where $R$ is the remainder including terms oscillating at other frequencies. The dispersive terms $D_{n}$ represent inverse Fourier transforms of the real propagation constant $k\left(\omega+\omega_{0}\right)$ expanded in power series around the reference wavenumbers $q_{n}$.

By grouping together terms oscillating at the same frequency we obtain:

$$
\begin{aligned}
& \frac{\partial A_{1}}{\partial z}+i D_{1} A_{1}=-i G(z) \frac{\chi^{(2)} \Omega}{2 n_{0} c}\left[A_{2} A_{1}^{*} e^{-i \Delta k_{1} z}+A_{3} A_{2}^{*} e^{-i \Delta k_{2} z}\right] \\
& \frac{\partial A_{2}}{\partial z}+i D_{2} A_{2}=-i G(z) \frac{\chi^{(2)} \Omega}{2 n_{0} c}\left[A_{1}^{2} e^{i \Delta k_{1} z}+2 A_{3} A_{1}^{*} e^{-i \Delta k_{2} z}\right], \\
& \frac{\partial A_{3}}{\partial z}+i D_{3} A_{3}=-i G(z) 3 \frac{\chi^{(2)} \Omega}{2 n_{0} c}\left[A_{1} A_{2}^{*} e^{i \Delta k_{2} z}\right],
\end{aligned}
$$

where $\Delta k_{1}=q_{2}-2 q_{1}$ and $\Delta k_{2}=q_{3}-q_{2}-q_{1}$. Dispersive operators are usually truncated at second order, so we take $D_{n} \approx \tilde{D}_{n}=-i k_{1 n} \partial_{t}-1 / 2 k_{2 n} \partial_{t t}$. We can now expand the nonlinear coefficient modulation $G(z)$ in Fourier series truncated at the third spatial harmonic: $G(z) \approx \frac{2}{\pi} e^{i 2 \pi / \Lambda z}+\frac{2}{3 \pi} e^{i 2 \pi / \Lambda z \cdot 3}$ where $\Lambda$ is the QPM period. Usually in ferroelectric 
materials such as lithium niobate and lithium tantalate, the dispersion is such that first harmonic matches SHG and third harmonic matches THG, so it is possible to write:

$$
\begin{aligned}
& \frac{\partial A_{1}}{\partial z}+i \tilde{D}_{1} A_{1}=-i \sigma_{1} A_{2} A_{1}^{*} e^{-i \delta k_{1} z}-i \sigma_{3} A_{3} A_{2}^{*} e^{-i \delta k_{2} z} \\
& \frac{\partial A_{2}}{\partial z}+i \tilde{D}_{2} A_{2}=-i \sigma_{2} A_{1}^{2} e^{i \delta k_{1} z}-i \sigma_{4} A_{3} A_{1}^{*} e^{-i \delta k_{2} z} \\
& \frac{\partial A_{3}}{\partial z}+i \tilde{D}_{3} A_{3}=-i \sigma_{5} A_{1} A_{2}^{*} e^{i \delta k_{2} z}
\end{aligned}
$$

where $\delta k_{1}=\Delta k_{1}-2 \pi / \Lambda$ and $\delta k_{2}=\Delta k_{2}-2 \pi / \Lambda \cdot 3$ are the residual mismatches, $\sigma_{1,2}=$ $d_{e f f, I} \frac{\Omega}{n_{0} c}, \sigma_{j}=(j-2) d_{e f f, I I} \frac{\Omega}{n_{0} c}[j=3,4,5]$ and $d_{e f f, I}=\frac{\chi^{(2)}}{2} \frac{2}{\pi}, d_{e f f, I I}=\frac{\chi^{(2)}}{2} \frac{2}{3 \pi}$.

This system is the usual coupled wave equations describing cascading THG, as can be obtained by Maxwell's equations [5, 17]. It is worth noting that the derived equations do not depend on $\omega_{0}$.

In order to show the validity of our approach, we simulated the propagation of a femtosecond pulse in a $L=5 \mathrm{~mm}$ long periodically poled stoichiometric lithium tantalate sample (PPSLT). To model the refractive index dispersion we employed a Sellmeier model fitted from experimental data [18] (temperature $T=25^{\circ} \mathrm{C}$ ) and nonlinear coefficient $d_{33}=\chi^{(2)} / 2=10.6 \mathrm{pm} / \mathrm{V}$. In the numerical solution of Eq. (1) we inserted the exact dispersion relation $k(\omega)$. We assumed a first order quasi phase matching (QPM) grating, with a period $\Lambda=17.4 \mu \mathrm{m}$. We injected a $T=120 \mathrm{fs}$ FWHM long gaussian pulse, centered around $1400 \mathrm{~nm}$, with $I=20 \mathrm{GW} / \mathrm{cm}^{2}$ peak intensity. The corresponding residual phase mismatch is $\delta k_{1}=10000 \mathrm{~m}^{-1}$ and $\delta k_{2}=8002 \mathrm{~m}^{-1}$. In the simulation we set the reference frequency $\omega_{0}$ to be equal to the second harmonic of the input pulse: in this way the second harmonic is stationary in the reference frame $\left(z^{\prime}, \tau\right)$. The change of reference frame is introduced by the change of variables $z=z$ and $\tau=t-k_{1} z$ [10]. 
Figure 1 shows the amplitude of the electric field envelope. The two concurrent processes of second and third harmonic generation appear clearly. The crystal dispersion makes the fundamental frequency (FF), second harmonic (SH) and TH (third harmonic) pulses to separate each other during propagation. The FF pump depletion is significant with a conversion efficiency of $37 \%$ to second harmonic and $9.5 \%$ to third harmonic.

We then simulated the same set up with coupled wave model (7). We approximated dispersion up to second order, calculating the relevant parameters from the Sellmeier relation. For inverse group velocities we used the following values (in $s / m$ ) $k_{11}=7.2136 \cdot 10^{-9}$, $k_{12}=7.538 \cdot 10^{-9}, k_{13}=8.293 e \cdot 10^{-9} ;$ whereas for group velocity dispersion we used (in $\left.s^{2} / m\right) k_{21}=1.0408 \cdot 10^{-25}, k_{22}=3.7514 \cdot 10^{-25}, k_{23}=7.9607 \cdot 10^{-25}$.

To compare the results we filtered the envelope $A$ around FF, SH and TH: the outcome of the comparison is shown in Fig. 2. The agreement is almost perfect for fundamental and second harmonic, whereas small differences can be noted on third harmonic. This can be ascribed to the higher dispersion at short wavelength, that makes the truncation at second order in Eqs. (7) sightly inaccurate.

\section{Experimental results}

For the experimental observation of the cascading SHG and THG we chose a PPSLT crystal. The 25-mm-long (L) sample presents poling periods ranging from $\Lambda=17.7 \mu \mathrm{m}$ to $\Lambda=21 \mu \mathrm{m}$ separated by non-poled regions. At the temperature of $160^{\circ} \mathrm{C}$ used in the experiments these periods correspond to phase matching wavelengths from $1440 \mathrm{~nm}$ to $1560 \mathrm{~nm}$. The near-IR pulses are derived from a non-collinear optical parametric amplifier (NOPA) pumped by the second harmonic of an amplified Ti:sapphire laser system (500 $\mu J, 150 \mathrm{fs}, 1 \mathrm{kHz})$. The NOPA generates $70 \mathrm{~nm}$ nearly transform-limited pulses tunable in the near IR (1300-1500 $\mathrm{nm}$ ) with widths around 40 fs (FWHM in intensity) and energy up to $1 \mu J$. The pulses are 


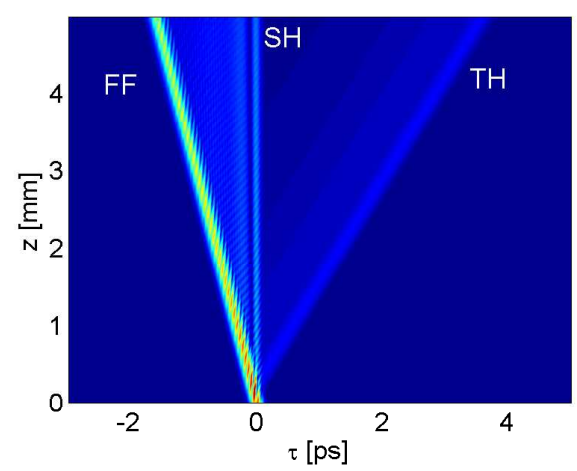

Fig. 1. Propagation of a femtosecond pulse in a PPLT crystal. a) Evolution of the field amplitude $|A|$ from numerical solution of Eq.(1). The initial pulse has gaussian shape and the parameters are $T=120 \mathrm{fs}, I=20 \mathrm{GW} / \mathrm{cm}^{2}, \lambda_{i n}=1400 \mathrm{~nm}, \lambda_{0}=2 \pi c / \omega_{0}=700 \mathrm{~nm}$, $d_{33}=\chi_{L T}^{(2)} / 2=10.6 p m / V$

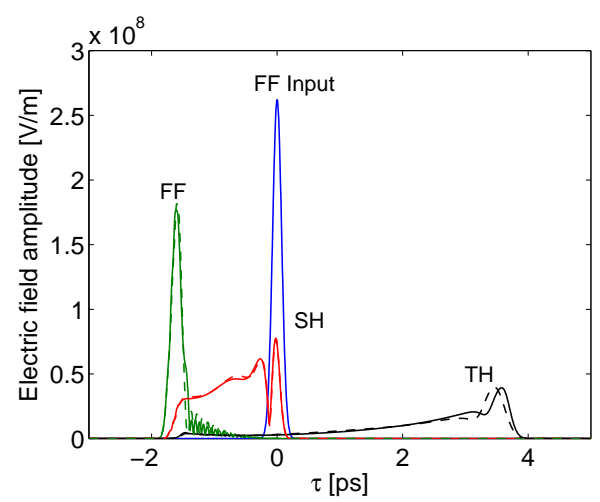

Fig. 2. Comparison between coupled wave solution (dashed curves) and $|A|$ filtered around fundamental, second harmonic and third harmonic (solid curves).

focused in the middle of the PPSLT crystal in order to obtain an effective interaction length of $2 \mathrm{~mm}$. This mild focusing enables to neglect both linear and nonlinear spatial effects. At the output of the sample the frequency spectra of the pulses are detected by a spectrometer.

Figure 3a) shows the outcome of the experiments performed with a FF pulse centered around $1390 \mathrm{~nm}$, with a peak intensity $\mathrm{I}=15 \mathrm{GW} / \mathrm{cm}^{2}$. The three periods $17.7 \mu \mathrm{m}, 18.1 \mu \mathrm{m}$ and $18.5 \mu \mathrm{m}$ phase match SH waves at $723.5 \mathrm{~nm}, 730.9 \mathrm{~nm}$ and $738 \mathrm{~nm}$, respectively. A discrete 

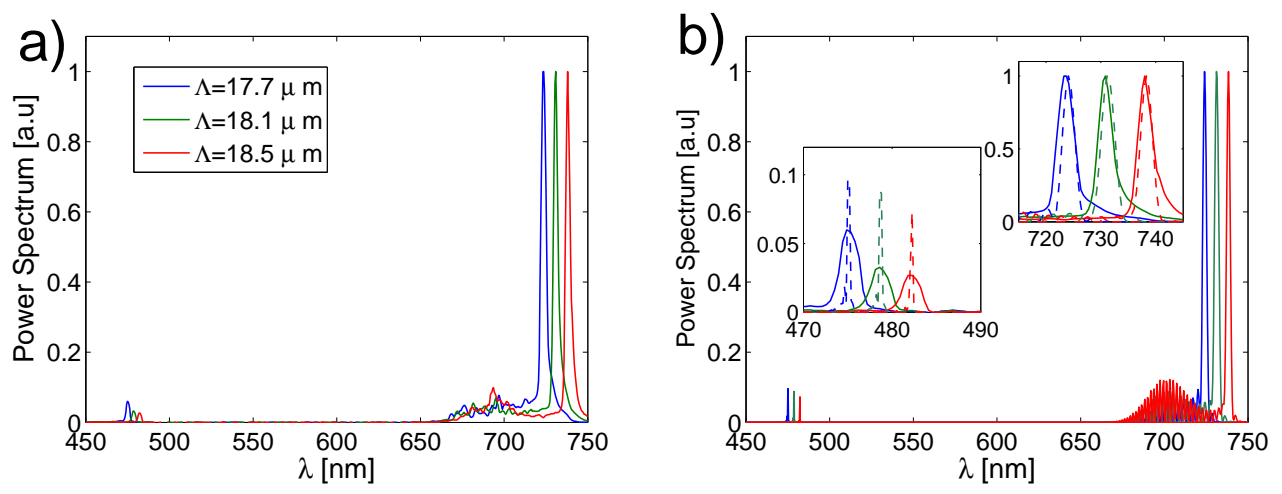

Fig. 3. Experimental (a) and numerical (b) SH and TH spectra at the crystal output for several poling periods. The inset in figure b) shows a close-up comparison between experiment and numerics at the second and third harmonic.

amount of non phase-matched second harmonic is visible between $650 \mathrm{~nm}$ and $720 \mathrm{~nm}$. We can also see the generation of third harmonic at $475 \mathrm{~nm}, 478.5 \mathrm{~nm}$ and $482 \mathrm{~nm}$, respectively. The amplitude of the $\mathrm{TH}$ peaks is around $5 \%$ of $\mathrm{SH}$ peaks.

Figure $3 \mathrm{~b}$ ) shows the results of the numerical solution of Eq. (1): we can see a good agreement with the experimental data. The two insets show the comparison between experiments (solid curves) and numerics (dashed curves) around the second and third harmonics. At the SH numerical and experimental curves are perfectly superimposed: peak positions and linewidths are perfectly captured by the theory. For what concerns third harmonic, we can see a perfect reproduction of the peak positions, but with a larger experimental linewidth. This discrepancy can be ascribed to the insufficient resolution of the spectrum analyzer used in the experiments.

\section{Conclusion}

We presented a model for the description of different types of parametric interactions in nonlinear optics which are associated with simultaneous phase-matching of several optical 
processes, based on a recently derived single wave boradband equation. This model is able to describe general quadratic nonlinear optical interactions and can be solved with modest computational efforts. We compared theoretical results with experiment on simultaneous second and third harmonic generation performed in periodically poled lithium tantalate crystals.

\section{References}

1. P. A. Franken, A. E. Hill, C. W. Peters, and G. Weinreich, "Generation of optical harmonics," Phys. Rev. Lett.7, 118-119 (1961).

2. R. W. Boyd, Nonlinear Optics, (Academic Press, 2003), 2nd ed.

3. M. M. Fejer, G. A. Magel, D. H. Jundt, and R. L. Byer, "Quasi-phasematched second harmonic generation: tuning and tolerances," IEEE J. Quantum Electron. 28,2631-2654 (1992).

4. M. Conforti, F. Baronio, and C. De Angelis, "From femtosecond infrared to picosecond visible pulses: temporal shaping with high-efficiency conversion, " Opt. Lett. 32, 1779$1781(2007)$.

5. S. M. Saltiel, A. A. Sukhourukov, and Y. S. Kivshar, "Multistep parametric processes in nonlinear optics," Prog. Optics 47, 1-73 (2005).

6. G. Z. Luo, S. N. Zhu, J. L. He, Y. Y. Zhu, H. T. Wang, Z. W. Liu, C. Zhang, and N. B. Ming, "Simultaneously efficient blue and red light generations in a periodically poled $L_{i} \mathrm{TaO}_{3}, "$ Appl. Phys. Lett. 78, 3006-3008 (2001).

7. V. Couderc, E. L. Lago, A. Barthelemy, C. De Angelis, and F. Gringoli, "Trapping of a weak probe through coupling with a two-color quadratic spatial soliton ," Opt. Commun. 203, 421-425 (2002).

8. A. A. Sukhorukov, T. J. Alexander, Y. S. Kivshar, and S. M. Saltiel, "Multistep 
cascading and fourth harmonic generation," Phys. Lett. A 281, 34-38 (2001).

9. M. C. Cardakli, D. Gurkan, S. A. Havstad, A. E. Willner, K. R. Parameswaran, M. M. Fejer, I. Brener, "Tunable all-optical time-slot-interchange and wavelength conversion using differencefrequency- generation and optical buffers," IEEE Photonics Technol. Lett. 14, 200-202 (2002).

10. M. Conforti, F. Baronio, and C. De Angelis, "Nonlinear envelope equation for broadband optical pulses in quadratic media," Phys. Rev. A 81, 053841(1-4) (2010).

11. M. Conforti, F. Baronio, and C. De Angelis, "Ultra-broadband optical phenomena in quadratic nonlinear media," IEEE Photonics J. 2, 600-610 (2010).

12. Sh. Amiranashvili, U. Bandelow, and A. Milke, "Pade approximant for the refractive index and nonlocal envelope equations," Opt. Commun. 283, 480-485 (2010).

13. T. Brabec and F. Krausz, "Nonlinear optical pulse propagation in the single-cycle regime," Phys. Rev. Lett. 78, 3282-3285 (1997).

14. M. Kolesik and J. V. Moloney, "Nonlinear optical pulse propagation simulation: From Maxwell's to unidirectional equations," Phys. Rev. E 70, 036604(1-11) (2004).

15. P. Kinsler, "Optical pulse propagation with minimal approximations," Phys. Rev. A 81, 013819(1-12) (2010).

16. A. V. Housakou and J. Herrmnann, "Supercontinuum generation of higher-order solitons by fission in photonic crystal fibers," Phys. Rev. Lett. 87, 203901(1-4) (2001).

17. Yi-Qiang Qin, Yong-Yuan Zhu, Chao Zhang, and Nai-Ben Ming, "Theoretical investigations of efficient cascaded third-harmonic generation in quasi-phase-matched and -mismatched configurations," J. Opt. Soc. Am. B 20, 73-82 (2003).

18. Ariel Bruner, David Eger, Moshe B. Oron, Pinhas Blau, and Moti Katz, "Temperature- 
dependent Sellmeier equation for the refractive index of stoichiometric lithium tantalate," Opt. Lett. 28, 194-196 (2003). 